\title{
Estudo epidemiológico dos casos de sífilis em gestantes em uma cidade do norte de Minas Gerais
}

\author{
Epidemiological study of syphilis cases in pregnant women in a city in the north of Minas \\ Gerais
}
Estudio epidemiológico de los casos de sífilis en gestantes en una ciudad del norte de Minas Gerais

Amanda Gonçalves Rocha e Araújo ${ }^{1}$, Lucas Faustino de Souza ${ }^{1}$, Nadine Antunes Teixeira1 ${ }^{1}$ Karoline de Souza Oliveira ${ }^{1}$, Joyce Micaelle Alves ${ }^{2}$, Claudia Mendes Campos Versiani ${ }^{1,2,3}$, Edilene Oliveira Amaral $^{1,3}$, Fernanda Cardoso Rocha ${ }^{1,2,3 *}$, Gregório Ribeiro de Andrade Neto ${ }^{1,3}$, Ana Paula de Oliveira Nascimento ${ }^{3}$, Leila das Graças Siqueira ${ }^{1,2}$

\section{RESUMO}

Objetivo: Descrever as características epidemiológicas maternas, dos casos notificados de sífilis em gestantes em uma cidade de médio porte da região norte de Minas Gerais no período compreendido entre de 2006 a 2016, segundo os dados do Sistema Nacional de Agravos de Notificação - SINAN. Método: Estudo descritivo e retrospectivo onde avaliou 124 fichas de notificação epidemiológica. As definições de casos seguiram as recomendações do Ministério da Saúde (MS) no Brasil, tendo as variáveis descritas utilizando se frequências relativa e absoluta. Resultados: As gestantes com sífilis, em maioria era jovens e estavam na faixa etária de 20 a 30 anos, com escolaridade menor ou igual ao ensino fundamental incompleto, sendo que $44,3 \%$ delas estavam no $3^{\circ}$ trimestre da gestação no momento da confirmação da doença. Todas realizaram testes laboratoriais, com predomínio do diagnóstico tardio. Conclusão: A realidade da sífilis em gestantes na cidade de Montes Claros (MG) está longe do esperado. Os resultados evidenciaram que as subnotificações é um crescente número de casos, em gestantes, elevando à persistência da transmissão vertical, evidenciando com isso a necessidade de ações voltadas para a infecção, assim como, uma política de controle da sífilis no município.

Palavras-chave: Cuidado pré-natal, Epidemiologia, Gestantes, Sífilis.

\begin{abstract}
Objective: To describe the maternal epidemiological characteristics of the reported cases of syphilis in pregnant women in a medium-sized city in the northern region of Minas Gerais in the period from 2006 to 2016, according to data from the National System of Notification Diseases - SINAN. Method: A descriptive and retrospective study evaluating 124 records of epidemiological notification. The definitions of cases followed the recommendations of the Ministry of Health (MS) in Brazil, with the variables described using relative and absolute frequencies. Results: Pregnant women with syphilis were mostly young and were between 20 and 30 years of age, with less than or equal to incomplete primary education, and $44.3 \%$ of them were in the third trimester of pregnancy at the time of confirmation disease. All of them underwent laboratory tests, with a predominance of late diagnosis. Conclusion: The reality of syphilis in pregnant women in the city of Montes

\footnotetext{
${ }^{1}$ Faculdades Unidas no Norte - FUNORTE. Montes Claros-MG. * E-mail: nandac.rocha@hotmail.com

2 Universidade Estadual de Montes Claros - UNIMONTES. Montes Claros-MG.

${ }^{3}$ Faculdades de Saúde Ibituruna - FASI. Montes Claros-MG.
} 
Claros (MG) is far from expected. The results showed that the underreporting is an increasing number of cases, in pregnant women, increasing the persistence of vertical transmission, thus evidencing the need for actions aimed at infection, as well as a syphilis control policy in the municipality.

Keywords: Prenatal care, Epidemiology, Pregnant women, Syphilis.

\section{RESUMEN}

Objetivo: Describir las características epidemiológicas maternas, de los casos notificados de sífilis en gestantes en una ciudad de tamaño mediano de la región del norte de Minas Gerais en el período comprendido entre 2006 a 2016, según los datos del Sistema Nacional de Agravios de Notificación - SINANIA. Método: Estudio descriptivo y retrospectivo donde evaluó 124 fichas de notificación epidemiológica. Las definiciones de casos siguieron las recomendaciones del Ministerio de Salud (MS) en Brasil, teniendo las variables descritas utilizando frecuencias relativa y absoluta. Resultados: Las gestantes con sífilis, en su mayoría eran jóvenes y estaban en el grupo de edad de 20 a 30 años, con escolaridad menor o igual a la enseñanza fundamental incompleta, siendo que el $44,3 \%$ de ellas estaban en el $3^{\circ}$ trimestre de la gestación en el momento de la confirmación de la gestación enfermedad. Todas realizaron pruebas de laboratorio, con predominio del diagnóstico tardío. Conclusión: La realidad de la sífilis en gestantes en la ciudad de Montes Claros (MG) está lejos de lo esperado. Los resultados evidenciaron que las subnotificaciones son un creciente número de casos en gestantes, elevando a la persistencia de la transmisión vertical, evidenciando con ello la necesidad de acciones dirigidas a la infección, así como, una política de control de la sífilis en el municipio.

Palabras clave: Cuidado prenatal, Epidemiología, Las mujeres embarazadas, La sífilis.

\section{INTRODUÇÃO}

A sífilis é transmitida, predominantemente, através de via sexual e transmissão vertical, sendo uma infecção de caráter sistêmico, causada pelo Treponema pallidum (T. pallidum), que acontece exclusivamente no ser humano e quando não tratada de forma precoce, pode evoluir para uma forma crônica com sequelas irreversíveis em longo prazo (BRASIL, 2015).

De acordo com pesquisas do Ministério da Saúde as Infecções Sexualmente Transmissíveis (IST's) são causadas por mais de 30 agentes etiológicos, podendo ser por vírus, bactérias, protozoários ou fungos e, são transmitidos principalmente por via sexual de forma eventual ou contato sanguíneo e no que se refere a sífilis essa infecção pode ainda acontecer na forma congênita, sendo transmitida da mãe para a criança durante a gestação, parto ou período de amamentação. Algumas destas IST's possuem alta taxa de incidência e prevalência facilitando a transmissão do HIV e trazendo complicações mais graves nas mulheres, causando cegueira, paralisia, alterações no sistema nervoso e cardiovascular, podendo levar a morte (BRASIL, 2015).

Recorre-se a Brasil (2015) para afirmar que vem ocorrendo aumento nos casos de sífilis em gestantes e sífilis congênita pelo mundo, pois, segundo estimativas da Organização Mundial de Saúde (OMS) (2013), mais de um milhão de pessoas adquirem uma IST diariamente. A cada ano, estima-se que 500 milhões de pessoas adquirem uma das IST curáveis (gonorreia, clamídia, sífilis e tricomoníase). Em relação aos casos notificados da doença no Brasil, ressalta-se que somente no ano de 2015 foram notificados 65.878 de casos de sífilis adquirida, dessas notificações cerca de 17.042 residiam na região sudeste do país. Na última década, observa-se um aumento de notificações para casos de Sífilis em gestantes, totalizando 33.365 no ano de 2015. Na série histórica dos casos de sífilis adquirida $60 \%$ das notificações pertencem ao sexo masculino, sendo que, no Brasil durante os anos de 2014 e 2015, a sífilis adquirida teve um aumento de $32,7 \%$, em gestantes $20,9 \%$ e $19 \%$ na forma congênita de sífilis (BRASIL, 2016).

Estudos de Lafetá et al. (2016), demostraram que em Minas Gerais, de acordo dados da Secretaria de Vigilância Epidemiológica, no ano de 2016 foram 5.245 casos de sífilis notificados no Sistema Nacional de 
Agravos de Notificação (SINAN). Reforça-se, ainda que, devido ao menor conhecimento sobre a sífilis, a maioria das pessoas transmite a seus parceiros durante o período assintomático da infecção. A sífilis quando não tratada pode evoluir para a forma mais grave, comprometendo o sistema nervoso e cardiovascular. Assim, os estágios da sífilis não tratada podem ser classificados como: sífilis primária, secundária, terciária e latente (BRASIL, 2015).

Na sífilis primária a manifestação principal é o cancro duro acompanhado de linfoadenomegalia regional, onde nem sempre os testes são reagentes, sífilis secundária as lesões na pele e mucosas se tornam mais evidentes e todos os testes reagentes detectam anticorpos, sífilis latente em que não se observam manifestações clínicas, mas os testes com anticorpos são reagentes, (recente até um ano após exposição e tardia com mais de um ano de evolução) e sífilis terciária que acomete não só a pele e mucosas, mas, também órgãos internos (BRASIL, 2016).

De acordo com Brasil (2015) a sífilis em gestantes é detectada através de exames durante o pré-natal realizados no $1^{\circ}$ trimestre e no $3^{\circ}$ trimestre por volta da $30^{\text {a }}$ semana de gestação, onde, quando detectada é necessário a intervenção imediata para reduzir a possibilidade de transmissão vertical, pois, dentre as principais complicações estão o aborto por sífilis e o óbito fetal, sendo classificada como precoce e tardia. Entretanto, na maioria das vezes, a sífilis é diagnosticada por rastreamento de gestantes ou de pessoas com IST. O diagnóstico da sífilis é feito através de exames laboratoriais. Para o diagnóstico pode-se utilizar os testes treponêmicos e os não treponêmicos. O Diagnóstico dependerá também da associação entre o histórico do indivíduo, os dados clínicos e a detecção de antígenos ou anticorpos por meio dos testes (BRASIL, 2010).

Fundamenta-se ainda em dados da OMS, em 2013, para descrever que cerca de 1,9 milhões de gestantes estavam infectadas pela sífilis, em todo o mundo. Além disso, nota-se ainda, que a sífilis tornou-se uma infecção reemergente, principalmente, em países da Europa, como a Itália e a América do Norte. E como a sífilis faz parte da lista de notificações compulsórias, ou seja, de caráter obrigatório, sua notificação pelos profissionais de saúde, conforme estabelece a Lei no 6259, de 30 de outubro de 1975 (BRASIL, 2010). E visando assegurar a mulher e à criança com direito ao nascimento seguro e o desenvolvimento e crescimento saudável, foi criada Rede cegonha como uma das ações complementares ao pré-natal. Dentre as ações da rede pode-se destacar a prevenção e tratamento das IST, HIV/Aids e Hepatites virais, disponibilizando também os testes rápidos para detectar a sífilis e HIV (BRASIL, 2014).

Este trabalho teve como objetivo descrever as características epidemiológicas maternas dos casos notificados de sífilis em gestantes em uma cidade de médio porte da região norte do Estado de Minas Gerais no período de 2006 a 2016 de acordo com a base de dados do Sistema Nacional de Agravos de Notificação - SINAN.

\section{MÉTODOS}

Trata-se de um estudo quantitativo, documental, retrospectivo e descritivo realizado a partir de informações disponíveis no banco de dados do Sistema de Informação de Agravos de Notificação (SINAN) do município de Montes Claros, cidade de médio porte localizada na região norte do estado de Minas Gerais e que possui área de 3.568,941 km² e contando com uma população estimada de 402.027 habitantes em 2017 (IBGE, 2017).

Foram incluídos todos os casos registrados de sífilis gestacional em mulheres gestantes residentes de Montes Claros, registrados no SINAN nos anos de 2006 a 2016, que atenderam ao critério de definição de caso confirmado segundo o Ministério da Saúde. Foram excluídos os casos cujas fichas encontravam-se incompletas ou indisponíveis para pesquisa ou casos duplicados e os casos não residentes no município identificado a partir da análise do SINAN. Foram estudadas as variáveis de acordo com as informações da ficha de notificação de sífilis em gestante, a saber: escolaridade, estado civil, faixa etária, diagnóstico e, classificação tratamento da gestante. 
Os dados foram coletados no mês de novembro de 2017, a partir da base de dados da vigilância epidemiológica desse município, que forneceu as informações em planilhas eletrônicas oriundas do SINAN referentes às notificações de sífilis em gestante no período pesquisado. Após os dados foram analisados descritivamente por meio do programa estatístico SPSS $\AA$, versão 20.0, para Windows e posteriormente foram comparados e discutidos de acordo com a literatura pesquisada e o olhar dos pesquisadores. $O$ estudo foi autorizado pelas instituições participantes e aprovado pelo Comitê de Ética em Pesquisa das Faculdades Unidas do Norte de Minas - FUNORTE, sob o parecer consubstanciado oㅡ 2.326.379, em 11 de outubro de 2017, e foi realizado em conformidade com a Resolução do Conselho Nacional de Saúde (CNS) № 466, de 12 de dezembro de 2012.

\section{RESULTADOS E DISCUSSÃO}

Os resultados refletem a vigilância quantitativa dos casos de sífilis na gestação realizada entre 2006 a 2016 em Montes Claros/MG de acordo com a base de dados do Sistema Nacional de Agravos de Notificação - SINAN, com o objetivo de descrever as características epidemiológicas de sífilis em gestantes.

Gráfico 1: Número de Casos de Sífilis em Gestantes notificados em Montes Claros/MG no período de 2006 a 2016.

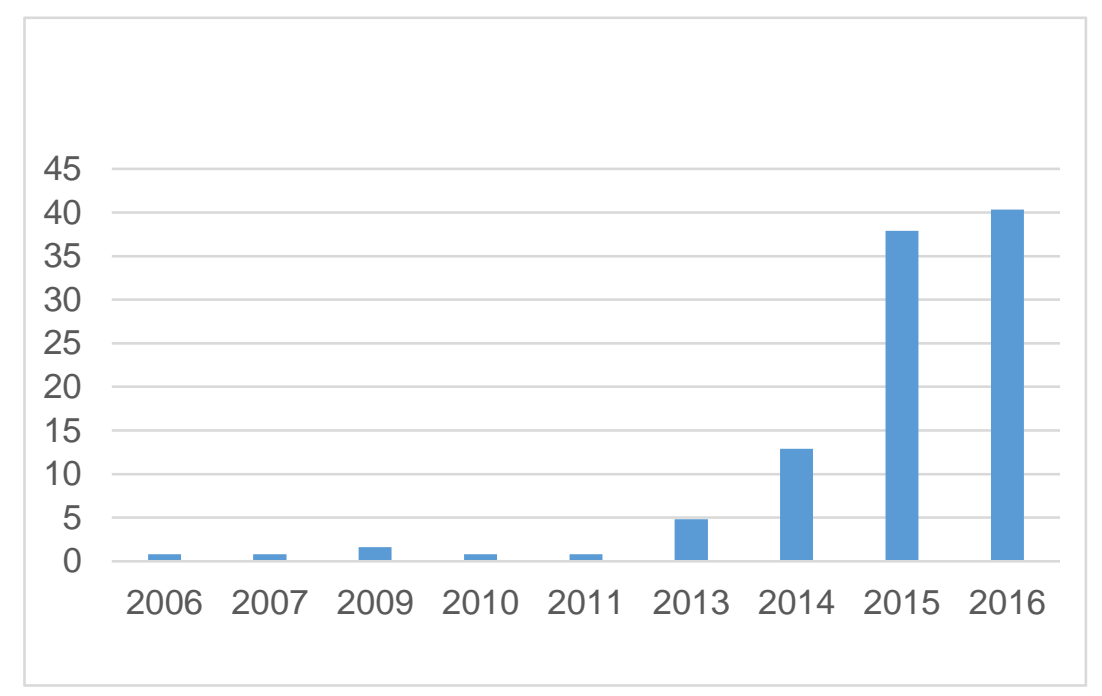

Fonte: SINAN 2006-2016 - SMS DE MONTES CLAROS/MG

Foram registrados 124 casos de sífilis em gestantes, no período entre 2006 a 2016, segundo dados do Sistema de Informação de Agravos de Notificação (SINAN) do setor de Vigilância Epidemiológica da Secretaria Municipal de Saúde de Montes Claros /MG. Constatou-se uma média de 12,4 casos novos por ano e ao calcular a incidência da sífilis em gestantes, construída a partir do número de casos detectados durante o ano para cada 1000 mil habitantes, identificou-se uma taxa de 0,31/1.000 habitantes. Com destaque para os anos de 2014, 2015 e 2016 com a maior taxa da doença (0,25/1.000 habitantes), apresentados no Gráfico $1 \mathrm{e}$, pode-se ainda, perceber o aumento significativo da doença no município.

O gráfico 1 permite afirmar que houve um aumento na incidência de gestantes com sífilis em Montes Claros/MG. Fato este que assemelha a vários outros municípios do Brasil, pois, esse aumento também tem sido registrado em outras cidades do país e do mundo conforme descreve estudos de Brasil (2015) e Bowen et al., (2015). Chama atenção as mulheres com sífilis que são, principalmente, jovens, residentes em zona rural, com baixa escolaridade, perfil esse semelhante ao apresentado em alguns estudos (CAMPOS, et al., 2010; LIMA, et al., 2013; CARVALHO; BRITO, 2014). 
Tabela 1. Características sociodemográficas de gestantes com sífilis no município de Montes Claros/MG de 2006 a 2016.

\begin{tabular}{|c|c|c|}
\hline Variáveis & no & $\%$ \\
\hline \multicolumn{3}{|c|}{ Idade (anos) } \\
\hline $10-20$ & 28 & 22.58 \\
\hline $20-30$ & 63 & 50,80 \\
\hline $30-40$ & 30 & 24,20 \\
\hline 40 ou + & 3 & 2,42 \\
\hline \multicolumn{3}{|c|}{ Escolaridade } \\
\hline Fundamental I & 29 & 23,39 \\
\hline Fundamental C & 11 & 8,87 \\
\hline Médio I & 17 & 13,71 \\
\hline Médio C & 28 & 22,58 \\
\hline Superior C & 3 & 2,42 \\
\hline Superior I & 1 & 0,81 \\
\hline Ignorado & 35 & 28,23 \\
\hline
\end{tabular}

A Tabela 1, acima apresentada, demonstra que a faixa etária das mulheres com sífilis maior notificada é a das mais jovens com idade entre 20 e 30 anos. Quanto à escolaridade a categoria ignorada teve um percentual de $28,23 \%$, seguido de ensino fundamental incompleto com $23,39 \%$.

Os dados acima descritos coincidem com os do estudo desenvolvido em Montes Claros/MG por Lafetá, et al. (2016), onde revelaram que os casos de sífilis estão na faixa etária predominante entre os 21 e 30 anos com $(50,8 \%)$, salientando-se que todos os casos notificados foram encontrados nas demais fontes de informação. Assim, recorre-se também a estudo desenvolvido por Rocha et al. (2016), onde foi possível identificar em que as gestantes acometidas por esta infecção são adolescentes e adultas jovens e, residentes na zona urbana. Dados semelhantes também foram encontrados em estudos de Coelho e Pinto (2009), onde descreveram que essa faixa etária de mulheres infectadas retrata que a prática da atividade sexual é muito precoce, e essa questão remete à ideia de que ainda são necessárias melhorias nas estratégias de educação em saúde nas escolas e unidades de saúde da família, visando conscientizar sobre a prática sexual segura.

Lafetá et al. (2016) destaca em seu estudo que a sífilis em gestantes permanece como doença a ser prevenida e controlada, no entanto, descreve que dificilmente consegue-se estabelecer um grupo de risco para a doença. Já outros estudos de Rodrigues e Guimarães (2004), Araújo et al (2012); Saraceni et al. (2005); LIMA et al. (2013) e Revollo (2007) descrevem características mais comuns nessa população, como predomínio da cor negra e parda, escolaridade inferior a 8 anos, idade de 20 a 29 anos, início da atividade sexual precoce e estado civil solteiro.

Outro dado descrito na Tabela 1 que chama atenção, refere-se a baixa escolaridade, considerada um marcador de maior risco para exposição às doenças sexualmente transmissíveis, devido a um limitado 
entendimento acerca das medidas de prevenção conforme afirma Serafim et al. (2014). Destaca-se que na presente pesquisa, verificou-se que a maioria das gestantes possuíam baixa escolaridade. Este achado assemelha-se ao de uma pesquisa realizada entre 2010 e 2013, em Belo Horizonte, capital do estado de Minas Gerais por NONATO; MELO e GUIMARAES, (2015). O mesmo resultado foi contatado em outros estudos que confirmam maior ocorrência de casos de sífilis em gestantes com baixa escolaridade (GONZALEZ; TAPIA; SERRUYA, 2014; LIMA, et al., 2013; SÁ, et al., 2001).

Guinsburg e Santos (2010) em um documento, referir-se o uso de drogas e o abandono da escola ao baixo nível socioeconômico, como fatores associados. Todavia, Almeida et al. (2009) destacaram um novo perfil de mulheres contaminadas, com maior escolaridade (mais que oito anos de ensino), casadas e com relato de apenas um parceiro sexual, os autores observaram em nos seus achados, diferentemente de alguns estudos brasileiros, predomínio de mulheres com escolaridade superior a 8 anos; com maioria se denominam parda, com idade de 21 a 30 anos e solteira. Os dados do presente estudo corroboram a favor de que a sífilis, assim como outras IST's, não afeta apenas um grupo de risco específico, devendo ser primordial a prevenção para a população geral.

Gráfico 2: Diagnóstico dos Casos de Sífilis em Gestantes, notificados por trimestre gestacional em Montes Claros/MG no período de 2006 a 2016.

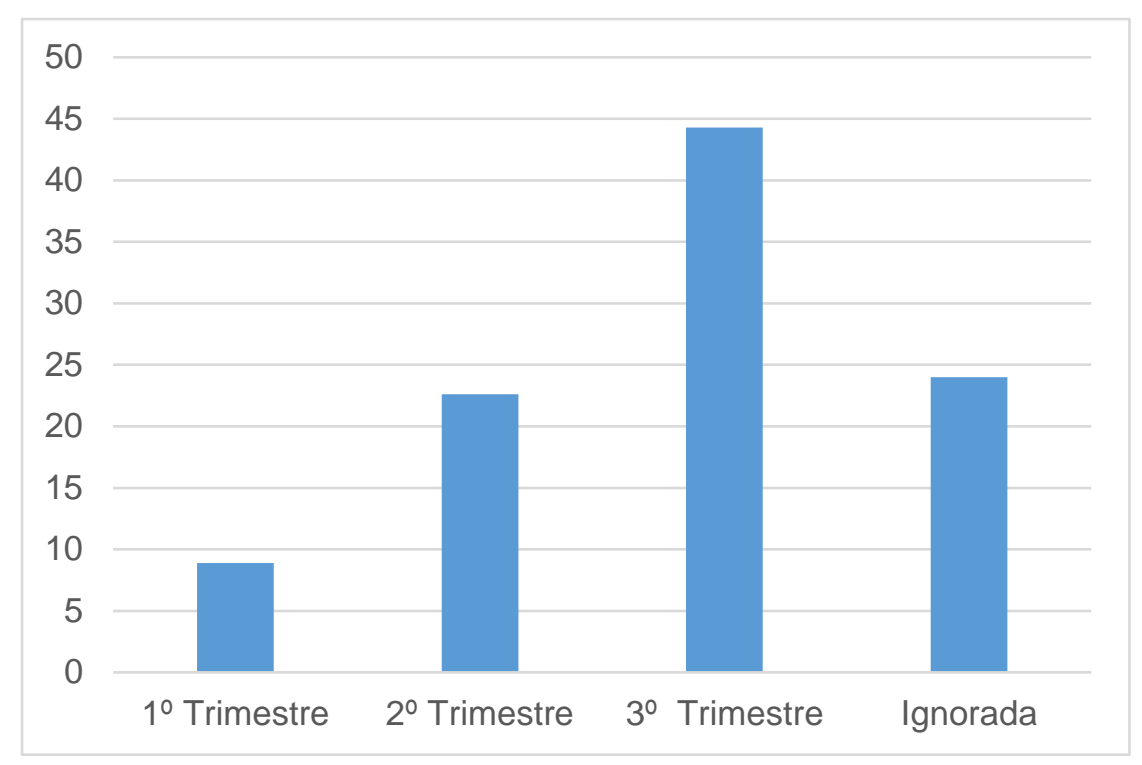

Fonte: SINAN 2006-2016 - SMS DE MONTES CLAROS/MG

O Gráfico 2 permite observar uma elevada frequência do diagnóstico de sífilis no $2^{\circ}$ e $3^{\circ}$ trimestres da gestação, conforme estudos de Araújo et al. (2008) a doença está presente nesse momento possivelmente devido ao início tardio do pré-natal que é quando as mesmas procuram pelo início do atendimento, levando assim à baixa sensibilidade e qualidade da assistência à gestante. Esses resultados ressaltam a importância da detecção oportuna das gestantes com sífilis, assim como, da oferta de oportunidades de tratamento correto para as gestantes e seus parceiros precocemente: a assistência pré-natal adequada é fundamental para a saúde materno-infantil e consequentemente, para a redução da sífilis congênita.

Os dados do estudo confirmam os resultados de Lafetá et al. (2016), onde também encontraram para o referido município pesquisado que, a maioria das gestantes apresentou diagnóstico tardio, ou seja, o mesmo ocorreu no momento do parto ou da curetagem (62,4\%). Assim, os resultados apresentados no Gráfico 2 levam à constatação da fragilidade dos serviços de saúde quanto ao controle da sífilis em gestantes e em 
seus RNs/congênita, pois o diagnóstico tardio leva a entender que ocorre o não tratamento adequado das mães e de seus parceiros.

Fundamenta-se em estudos de Saraceni et al., (2007) para reforçar a importância do VDRL (Veneral Disease Research Laboratory) ser realizado no primeiro trimestre da gravidez e de repeti-lo no terceiro trimestre. Ainda, de acordo o Ministério da Saúde, há uma grande dificuldade no diagnóstico clínico de sífilis em gestantes, visto que lesões como o cancro duro não causam sintomas e geralmente, estão localizadas em locais de difícil visualização como: parede vaginal, cérvix ou períneo (BRASIL, 2015). Ressalta-se que erros de interpretação e classificação da fase clínica da sífilis podem levar a tratamentos inadequados e, que devem ser classificados como sífilis de duração ignorada todos os casos que não é possível estabelecer a evolução da doença e naqueles com ausência de sinais clínicos, sendo preconizado o tratamento desses casos com três doses de penicilina benzatina (BRASIL, 2015).

Gráfico 3: Classificação dos casos de Sífilis em Gestantes, notificados por fase clínica em Montes Claros/MG no período de 2006 a 2016.

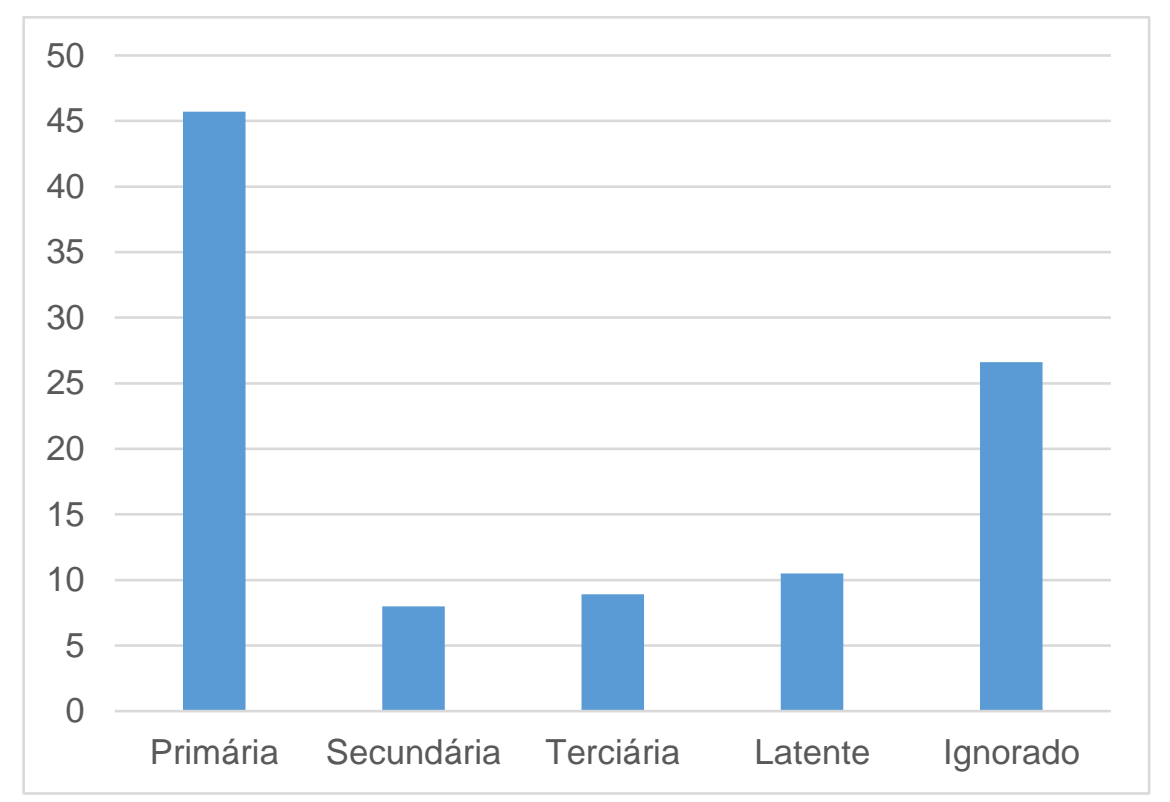

Fonte: SINAN 2006-2016 - SMS DE MONTES CLAROS/MG

No presente estudo foi possível evidenciar que a maioria das gestantes tiveram a classificação para sífilis primária, o que condiz com estudos desenvolvidos por Lafetá et al (2016). E, chamam atenção, para o fato de que este resultado poderia ter o número de ignorados a reduzidos desde que cada gestante cumprisse com o mínimo recomendado de seis consultas de pré-natal, com início no primeiro trimestre. Mas, como ainda persiste o diagnóstico tardio ou ignorado o controle da sífilis em gestantes e em seus RNs fica ainda mais difícil. Além disso, vale lembrar que todos os RNs de gestantes com sífilis devem ser investigados para sífilis congênita.

Além da realidade anteriormente discutida, os dados do SINAN mostraram que as fichas de notificação apresentam falhas em alguns registros, com informações ausentes e incoerentes. Um exemplo a ser destacado é a inconsistência de datas, ou seja, os dados referentes à data de realização do diagnóstico, notificação e classificação dos casos se diferem muito. Assim, é primordial uma discussão sobre a necessidade da melhoria no registro dessas informações nas fichas de notificação, uma vez que, elas darão subsídios às ações de controle, monitoramento e prevenção da doença. 


\section{CONCLUSÃO}

No período de 2006 a 2016, foram notificados 124 casos de sífilis em gestantes, concluindo que as gestantes com sífilis, a maioria era jovem e estava na faixa etária de 20 a 30 anos e com escolaridade menor ou igual ao ensino fundamental incompleto, sendo que $44,3 \%$ delas estavam no $3^{\circ}$ trimestre da gestação no momento que tiveram a confirmação da sífilis.

A realidade da sífilis na cidade está longe do que se espera. Há subnotificação de casos, aumento progressivo da doença nas gestantes e constância de transmissão vertical, o que sinaliza ausência de eficácia nas políticas de controle da sífilis no município estudado. Partindo do pressuposto que a atenção básica é a principal porta de entrada aos serviços de saúde, sendo o elo mais próximo entre cliente e profissionais, é pertinente a adoção de medidas para o controle da doença visando alterar o cenário epidemiológico da sífilis.

E, por basear-se em dados secundários, é possível identificar limitações inerentes a este tipo de pesquisa, que objetivou descrever o perfil dessa população. Sendo assim, recomenda-se que novos estudos sobre esta temática sejam realizados em nossa região, de modo a aprofundar os conhecimentos voltados para a necessidade de saúde dos pacientes com sífilis em especial os RNs destas gestantes.

\section{AGRADECIMENTO}

Nosso agradecimento ao Programa de Iniciação Científica (PROIC) das Faculdades Unidas do Norte Minas - FUNORTE por toda a contribuição e apoio.

\section{REFERÊNCIAS}

1. ALMEIDA KC, et al. Sífilis em gestantes atendidas em uma unidade de saúde pública de Anápolis, Goiás, Brasil. RBCA; 2009, 41(3): 1814, 2009.

2. ARAÚJO CL, et al. Incidência da sífilis congênita no Brasil e sua relação com a Estratégia Saúde da Família. Rev Saúde Pública 2012; 46(3): 479-486.

3. ARAÚJO MAL, et al. Análise da qualidade dos registros nos prontuários de gestantes com exame de VDRL reagente. Rev APS. 2008, 11(1):4-9.

4. BOWEN V et al. Increase in incidence of congenital syphilis: United States of America, 2012-2014. MMWR Morb Mortal Wkly Rep. 2015, 64(44): 1241-5.

5. BRASIL. Ministério da saúde. Conselho nacional de saúde. Resolução no 466, de 12 de dezembro de 2012. Diário Oficial da União, 2013.

6. BRASIL. Ministério da Saúde. Coordenação de Doenças Sexualmente Transmissíveis e Aids. Sífilis: Estratégias para Diagnóstico no Brasil. Brasília. 2010.100 p.

7. BRASIL. Ministério da saúde. Departamento de vigilância epidemiológica. Secretaria de vigilância em saúde. Sistema de Informação de Agravos de Notificação-Sinan: normas e rotinas. Brasília. 2007.

8. BRASIL. Ministério da saúde. Secretaria de vigilância em saúde. Departamento de DST, aids e hepatites virais. Protocolo clínico e diretrizes terapêuticas para atenção integral às pessoas com infecções sexualmente transmissíveis. 2015. 120p.

9. BRASIL. Ministério da saúde. Secretaria de vigilância em saúde. Departamento de DST, aids e hepatites virais. Manual Técnico para Diagnóstico da Sífilis/Prevenção e Controle das Doenças Sexualmente Transmissíveis, Aids e Hepatites Virais. 2016. 52p.

10. BRASIL. Ministério da saúde. Transmissão vertical do HIV e Sífilis: Estratégias para Redução e Eliminação. Brasília. 2014. 20p.

11. CAMPOS ALA, et al. Epidemiologia da sífilis gestacional em Fortaleza, Ceará, Brasil: um agravo sem controle. Cad Saude Publica. 2010, 26(9):1747-55.

12. CARVALHO IS, BRITO RS. Sífilis congênita no Rio Grande do Norte: estudo descritivo do período $2007-2010$. Epidemiol Serv Saude. 2014,13(2):287-94.

13. COELHO APF, PINTO, MJFS. Ocorrência de Sífilis em Mulheres Atendidas em um Centro de Testagem e Aconselhamento de São Luís. São Luís-MA, 2009. 
14. COELHO, Nivaldo. Ministério da Saúde lança ação nacional de combate à sífilis. Portal da saúde. Ministério da Saúde. Brasília, 20 de out. 2016.

15. GONZALES GF, et al. Sífilis gestacional y factores asociados en hospitales públicos del Perú en el periodo 20002010. Rev Peru Med Exp Salud Publica, 2014, 31(2): 211-21.

16. GUINSBURG, R; SANTOS, AMN. Critérios diagnósticos e tratamento da sífilis congênita. São Paulo: Departamento de Neonatologia, Sociedade Brasileira de Pediatria; 2010.

17. IBGE-CIDADES. Instituto Brasileiro de Geografia e Estatística.

18. LAFETÁ KRG, et al. Sífilis materna e congênita, subnotificação e difícil controle. Rev. bras. epidemiol, 2016,19(1):63-74.

19. LIMA MG et al. Incidência e fatores de risco para sífilis congênita em Belo Horizonte, Minas Gerais, $2001-2008$. Ciênc Saúde Coletiva; 2013, 18(2):499-506.

20. NONATO SM, et al. MELO, APS; GUIMARÃES, MDC. Sífilis na gestação e fatores associados à sífilis congênita em Belo Horizonte-MG, 2010-2013. Epidemiol Serv Saude, 2015, 24(4): 681-94.

21. REVOLLO R. et al. Maternal and congenital syphilis four provinces in Bolivia. Salud Publica Mex; 2007, 49(6):422-8.

22. ROCHA, RPS. et al. Análise do perfil epidemiológico de sífilis nas gestantes e crianças, em Tangará da Serra, de 2007 a 2014. Revista Norte Mineira de Enfermagem, Montes Claros,2016,5(2):03-21.

23. RODRIGUES, CS; GUIMARÃES MDC. Grupo Nacional de Estudo sobre Sífilis Congênita. Positividade para sífilis em puérperas: ainda um desafio para o Brasil. Rev Panam Salud Publica, 2004,16(3):168-75.

24. SÁ RAM et al. Sífilis e gravidez: avaliação da prevalência e fatores de risco nas gestantes atendidas na Maternidade Escola - UFRJ. DST -J Bras Doencas Sex Transm, 2001,13(4): 6-8.

25. SARACENI V, et al. Mortalidade perinatal por sífilis congênita: indicador da qualidade da atenção à mulher e à criança. Cad Saúde Pública; 2005,21(4):1244-50.

26. SARACENI V, et al. Vigilância da sífilis na gravidez. Revista Epidemiologia e Serviços de Saúde;2007,16( 2):103 $-111$.

27. SERAFIM AS et al. Incidence of congenital syphilis in the South Region of Brazil. Rev Soc Bras Med Trop.2014, 47( 2):170-8, World Health Organization. Investment case for eliminating mother-to-child transmission of syphillis: promoting better maternal and child health and stronger health systems. Geneva: World Health Organization; 2013. 